\title{
Trends in the lifetime risk of COPD exacerbation requiring hospitalisation
}

\author{
Jesper Lykkegaard ${ }^{1}$, René dePont Christensen ${ }^{1}$, Jesper Rømhild Davidsen ${ }^{1,2}$, \\ Henrik Støvring ${ }^{3}$, Morten Andersen ${ }^{1,4}$ and Jens Søndergaard ${ }^{1}$
}

\author{
Affiliations: \\ ${ }^{1}$ Research Unit of General Practice, Institute of Public Health, University of Southern Denmark, Odense, \\ ${ }^{2}$ Dept of Respiratory Medicine, Odense University Hospital, Odense, and \\ ${ }^{3}$ Dept of Public Health, Biostatistics, Aarhus University, Aarhus, Denmark. \\ ${ }^{4}$ Centre for Pharmacoepidemiology, Karolinska Institutet, Karolinska University Hospital, Stockholm, Sweden.
}

\section{Correspondence:}

J. Lykkegaard, University of Southern Denmark, Faculty of Health Sciences, Institute of Public Health, Research Unit of General Practice, J. B. Winsløws Vej 9A, DK-5000 Odense C, Denmark.

E-mail: jlykkegaardahealth.sdu.dk

ABSTRACT This study aimed to estimate time trends in the lifetime risk of hospitalisation with exacerbation of chronic obstructive pulmonary disease (COPD) in Denmark.

During the period from 1994 to 2008, a register-based cohort study was conducted covering each subject in the entire population of Denmark (5.18 million in 2008). Based on previously validated diagnosis codes, all COPD hospitalisations were identified. Individual retrospective review periods of 8 years were used to determine first-time hospitalisations. From year 2002 to 2008, all first-time COPD hospitalised subjects aged $\geqslant 30$ years were identified. The calculation of lifetime risk was based on age- and sex-specific first-time COPD hospitalisation rates and rates of COPD hospitalisation-free survival, assuming them to be calendar time stationary.

The study included 23.9 million person-years of risk time and identified 48959 first-time COPD hospitalisations. For 30-year-olds in Denmark, the lifetime risk of COPD hospitalisation was 12.0\% (95\% CI 11.9-12.2) for females and $10.9 \%$ (95\% CI 10.8-11.1) for males. Trends were generally equal in both sexes.

During the period from 2002 to 2008, the rate of first-time COPD hospitalisations decreased, while the survival of never COPD hospitalised subjects increased. In consequence, the lifetime risk of COPD hospitalisation remained constant.

@ERSpublications

A $10 \%$ lifetime risk of COPD hospitalisation remains constant http://ow.ly/mxsU0

Received: Aug 172012 | Accepted after revision: Jan 222013 | First published online: Feb 212013

Support statement: The study was supported by a research fellowship to Jesper Lykkegaard from the University of Southern Denmark, and a research grant from the Governmental Region of Southern Denmark.

Conflict of interest: Disclosures can be found alongside the online version of this article at www.erj.ersjournals.com

Copyright @ERS 2013 


\section{Introduction}

Chronic obstructive pulmonary disease (COPD) is a preventable disease, but the exposure to inhaled gases and particles, e.g. tobacco smoke, which is the major cause of the disease, usually begins decades before symptomatic disease can be detected [1]. Because of this temporal detachment of cause and effect and because not all smokers live to develop the disease, risk estimates of developing the disease are important to individual preventive counselling. Also, for healthcare planners, risk estimates are needed to anticipate its future burden and to prioritise prevention resources between COPD and other preventable health risks.

Risk is preferably estimated as the age-specific incidence rates. However, the full spectrum of age-specific incidence rates is difficult to communicate to patients and politicians, and also to compare across countries and diseases. Furthermore, the risk of developing a chronic disease, such as COPD, at some future age (the age-specific incidence rate) provides little information to a patient, if the chances of surviving until that age without already having developed the disease are not considered.

Lifetime risk estimates overcome this problem by summing up the spectrum of age-specific incidence rates and the rates of disease-free survival into one single number $[2,3]$. Such estimates are commonly used benchmarks with regard to other chronic diseases, such as cardiovascular disease and cancer, but they have not until recently been adopted in COPD $[4,5]$.

The few previous COPD lifetime risk studies [6-8] have not focused on the risk of developing the end stages of COPD, in which mortality, quality of life reduction and healthcare costs are highest, and hospitalisations with exacerbation of the disease are frequent [9-12].

This study aimed to estimate time trends in the lifetime risk of hospitalisation with COPD in Denmark.

\section{Methods \\ Design}

During the period from January 1, 1994 to December 31, 2008, a register-based cohort study was conducted covering each subject in the entire population of Denmark (5.18 million in 2008 [13]).

\section{Data sources}

In Denmark, healthcare services are in general free of charge and with equal access for all citizens. COPD hospitalisations are to public hospitals and occur either by referral from a general practitioner or, in severe cases, as direct emergency admissions.

Since 1977, the Danish National Patient Registry has recorded administrative data from all Danish admissions to hospital [14]. From 1994 and onwards, diagnoses are classified according to the International Classification of Diseases 10th revision (ICD-10) [15]. All data are registered with the patient's unique civil registration number, which allows data linkage on an individual level between all national registers [16].

We identified all hospitalisations with COPD in Denmark during the years 1994 to 2008, and linked the individual health administrative data with data from the Demographic Register regarding the patients' dates of birth, death, and migrations to or from Denmark. For the years 2002 to 2008, the Demographic Register also provided age- and sex-specific numbers of subjects and deaths in the total Danish population.

\section{Definition of hospitalisation with COPD}

COPD hospitalisation was defined as any hospitalisation with either ICD-10 codes J41-44 (COPD, chronic bronchitis or emphysema) as primary diagnosis or with J13-18 (pneumonia) or J96 (respiratory failure) as primary diagnosis combined with $\mathrm{J} 41-44$ as a secondary diagnosis.

Except for the inclusion of J41-43, the aforementioned combinations of diagnosis codes are used in the Danish healthcare system to monitor and pay for the hospital treatment of COPD, and a 2008 nationwide validation study found a $93 \%$ positive predictive value for J44 as primary diagnosis and $87 \%$ as secondary to respiratory failure or pneumonia [14]. The inclusion of J41-43 (chronic bronchitis and emphysema) was suggested in the validation paper to obtain the best possible sensitivity and specificity. In a prior study, we showed that hospitalisations coded with J41-43 did not significantly differ from those coded with J44 (COPD) [17]. Sensitivity analyses leaving out pneumonia as primary diagnosis and exclusively including J44 as primary diagnosis were conducted.

\section{Definition of incident and prevalent cases}

First-time COPD hospitalisations (incident cases) were defined as any COPD hospitalisation of a subject who had not been hospitalised with COPD within a period of 8 years before the hospitalisation in question. Correspondingly, subjects were classified as prevalent cases between first COPD hospitalisation and death, migration, or the end of 8 years of individual follow-up with no COPD hospitalisations (whichever 
occurred first). The 7-year study period and the longer 8-year retrospective review period were chosen because ICD-10 classified data were only available from year 1994 to 2008, and we wanted both unbiased trend estimates and to avoid the possibility of the same subject being regarded as first-time hospitalised twice in our study period. Sensitivity analyses as to the length of the retrospective review period were performed, reducing it to 5 years in the overall estimate and increasing it to 14 years, which was only possible in year 2008.

\section{Estimation of lifetime risk}

On January 1 each year from 2002 to 2008 , all Danish subjects aged $\geqslant 30$ years were categorised as being either prevalent or at risk of first-time COPD hospitalisation. For each calendar year, all subjects at risk in the beginning of the year were further categorised as having either died or been hospitalised with COPD (whichever occurred first), or as remaining at risk throughout the year. Using 1-year age strata from age 30 years until $>99$ years, the age- and sex-specific number of subjects in each category was calculated for each calendar year. The incidence rates of first-time COPD hospitalisations and the mortality rates of subjects never hospitalised with COPD were estimated from the number of incident subjects and the number of subjects who died before COPD hospitalisation, respectively, divided by the number of subjects at risk in the beginning of each year.

The key to calculating the annual lifetime risk estimates is the assumption that in a future lifespan from age 30 years until $>99$ years, the above-mentioned age-specific incidence and mortality rates were to remain constant. With this assumption, a method described by KeIDING [2] was used to estimate the lifetime risk of COPD hospitalisation.

For each age from 30 years to $>99$ years, the probability of COPD hospitalisation-free survival from age 30 years until a given age was calculated as the product of the age-specific probabilities from age 30 years until that given age. The conditional probability of being hospitalised with COPD at a specific age, given COPD hospitalisation-free survival from age 30 years until that given age, was calculated as the product of the age-specific COPD-hospitalisation-free survival probability and the incidence rate of first-time COPD hospitalisation at the given age. Finally, the residual lifetime risk at a given age was calculated as the sum of the above conditional probabilities from that age until age $>99$ years. Confidence intervals were calculated using bootstrap techniques. All analyses were performed using STATA Release 11.0 (STATACorp, College Station, TX, USA).

We started the calculations at age 30 years because the diagnosis codes were validated from that age onwards and because COPD hospitalisations before that age are extremely rare. Hence, the few young adults that we identified with a COPD diagnosis in the register may instead have had asthma, just as the more frequent children aged 0-2 years also coded with COPD may very probably have had altogether different airway diseases.

The study was approved by the Danish Data Protection Agency (reference number 2009-41-3337). As the study was register-based, according to Danish legislation no approval from the Biomedical Research Ethics Committee was required.

\section{Results}

During the period from year 2002 to 2008 , the Danish population aged $\geqslant 30$ years increased from 3.39 to 3.51 million persons and the observed cumulated person risk time for first-time COPD hospitalisation was 23.9 million person years. We identified 22749 males with first-time COPD hospitalisations and 26210 females. The hospitalisations of patients who were aged $\geqslant 45$ years $(97.5 \%)$ were characterised in a prior paper; inpatient mortality increased from 5.9 to $7.0 \%$ and 1-year mortality from 23.2 to $25.9 \%$ [17].

Among Danes aged $\geqslant 30$ years, the incidence rate of first-time COPD hospitalisations was 2.14 per 1000 person years for females (95\% CI 2.11-2.16) and 1.95 for males (95\% CI 1.93-1.98) (table 1). The risk of first-time COPD hospitalisation increased with age until $\sim 80$ years for females and 85 years for males. Above these ages, the risk decreased with increasing age (fig. 1 and table 1).

During the period from year 2002 to 2008, in both sexes, the residual lifetime risk for 30-year-olds to be hospitalised with COPD remained roughly constant. Therefore, no further trend analyses were made. Based on the total period, using the main case definition, the risk was $12.0 \%$ (95\% CI 11.9-12.2) for females and $10.9 \%$ (95\% CI 10.8-11.1) for males. Using the strictest case definition, exclusively J44 as primary diagnosis, the risk was reduced to $9.6 \%$ in females and $8.4 \%$ in males (table 2).

The residual lifetime risk for 30-year-olds consisted primarily of the conditional probabilities between 45 and 90 years of age (fig. 2). This was because of low rates of first-time hospitalisation and hospitalisationfree survival, respectively, below and above this age interval. 
TABLE 1 Age- and sex-specific rates of first-time chronic obstructive pulmonary disease (COPD) hospitalisations in Denmark during 2002-2008; main case definition

\begin{tabular}{|c|c|c|c|c|c|c|c|c|}
\hline Age years & \multicolumn{4}{|c|}{ Females } & \multicolumn{4}{|c|}{ Males } \\
\hline $30-39$ & 293 & 2735642 & 0.11 & $0.09-0.12$ & 222 & 2811255 & 0.08 & $0.07-0.09$ \\
\hline $40-49$ & 1107 & 2667380 & 0.41 & $0.39-0.44$ & 860 & 2744868 & 0.31 & $0.29-0.33$ \\
\hline 70-79 & 9201 & 1325121 & 6.94 & $6.80-7.08$ & 8255 & 1068412 & 7.73 & $7.56-7.89$ \\
\hline $80-89$ & 5824 & 807149 & 7.16 & $6.98-7.35$ & 5236 & 443690 & 11.80 & $11.48-12.12$ \\
\hline$\geqslant 90$ & 790 & 178549 & 4.42 & $4.12-4.73$ & 510 & 56593 & 9.01 & $8.23-9.79$ \\
\hline Total & 26210 & 12274932 & 2.14 & $2.11-2.16$ & 22749 & 11642234 & 1.95 & $1.93-1.98$ \\
\hline
\end{tabular}

${ }^{\#}$ : person years at risk of first-time COPD hospitalisation; ${ }^{\circ}$ : per 1000 person years.

From age 30 years, for females, the residual lifetime risk continuously decreased with increasing age. For males, the residual lifetime risk increased slightly until age 48 years, after which it decreased. Until age 65 years, the risk was higher in females, but above that age, the male risk was higher (fig. 3 and table 3 ).

Extending the retrospective review period for the analysis of year 2008 from 8 to 14 years did not lower the lifetime risk estimates substantially, while decreasing the retrospective review period to 5 years in the overall analysis increased the lifetime risk estimate for males from $10.9 \%$ (95\% CI $10.8-11.1$ ) to $11.6 \%$ (95\% CI $11.4-11.7)$ and for females from $12.0 \%(95 \%$ CI $11.9-12.2)$ to $12.8 \%$ (95\% CI $12.7-13.0)$.

\section{Discussion}

This is the first study to estimate the lifetime risk of COPD hospitalisation. Data from 15 years of continuous and complete coverage of all 5.43 million citizens of Denmark were analysed and the main observation is that, for 30 -year-old Danes, the lifetime risk of having a COPD exacerbation requiring hospitalisation is $12 \%$ for females and $11 \%$ for males. Considering the $25 \% 1$-year mortality and the fact that multiple minor to moderate exacerbations as well as many years of progressive symptoms and reduced quality of life usually precedes hospitalisation with $\operatorname{COPD}[12,18]$, this study confirms that COPD is among the most important public health problems.

Three prior COPD studies have reported lifetime risks in the general population. In Ontario, Canada, using 14 years worth of validated register data from both primary and secondary care on $\sim 6$ million subjects, GERSHON et al. [6] reported a 26\% female and 30\% male risk of developing physician-diagnosed COPD. In a similar Dutch study including 185000 subjects in 400 general practices spread across the country, AFONSO et al. [8] reported a substantially lower $8.3 \%$ female and $12.7 \%$ male risk. In that study, however, all records of potential COPD underwent expert review using a somewhat stricter case definition. In contrast to the

FIGURE 1 Risk of first-time chronic obstructive pulmonary disease hospitalisation in 1 year.

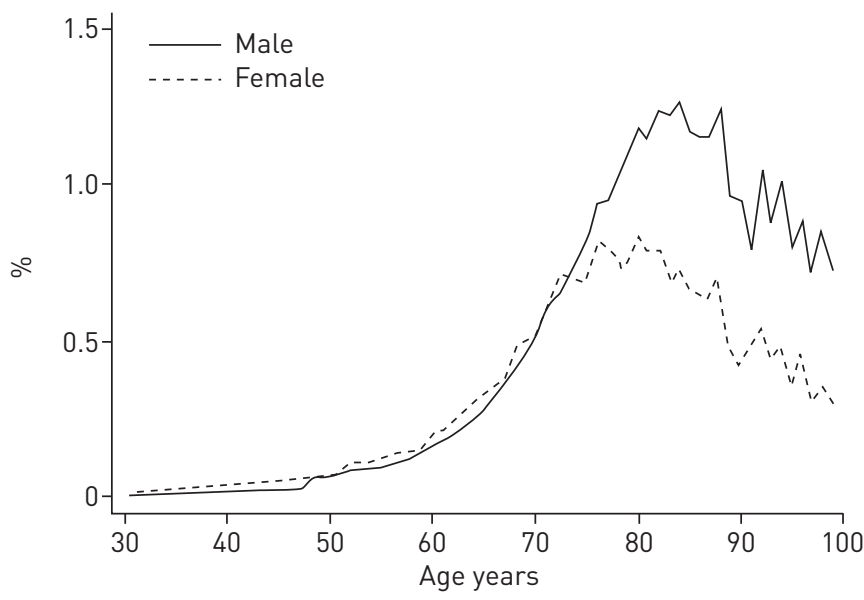


TABLE 2 Residual lifetime risk of chronic obstructive pulmonary disease hospitalisation for 30-year-olds in Denmark during the period 2002-2008

\begin{tabular}{|c|c|c|c|c|c|c|}
\hline \multirow[t]{2}{*}{ Period } & \multicolumn{3}{|c|}{ Females } & \multicolumn{3}{|c|}{ Males } \\
\hline & Main definition & Without pneumonia & Exclusively J44 & Main definition & Without pneumonia & Exclusively J44 \\
\hline 2003 & $11.9(10.5-13.4)$ & $10.7(9.3-12.1)$ & $9.5(6.6-12.5)$ & $11.1(10.3-11.9)$ & $9.6(8.6-10.6)$ & $8.5(7.0-9.9)$ \\
\hline 2004 & $11.7(11.0-12.5)$ & $10.4(8.9-11.9)$ & $9.3(7.8-10.9)$ & $10.8(9.7-12.0)$ & $9.4(8.0-10.7)$ & $8.2(7.0-9.5)$ \\
\hline 2005 & $12.4(10.7-14.0)$ & $11.1(9.6-12.5)$ & $9.9(7.2-12.6)$ & $10.9(9.5-12.3)$ & $9.4(7.8-10.9)$ & $8.5(6.8-10.2)$ \\
\hline 2008 & $12.3(11.3-13.3)$ & $10.9(9.0-12.8)$ & $9.9(8.0-11.7)$ & $10.7(9.8-11.7)$ & $9.2(8.6-9.8)$ & $8.0(6.7-9.3)$ \\
\hline Pooled & $12.0(11.9-12.2)$ & $10.7(10.6-10.9)$ & $9.6(9.5-9.7)$ & $10.9(10.8-11.1)$ & $9.4(9.3-9.6)$ & $8.4(8.2-8.5)$ \\
\hline
\end{tabular}

Data are presented as \% risk $(95 \% \mathrm{CI})$.

register-based studies of physician-diagnosed COPD, VAN DURME et al. [7] conducted an 11-year cohort study. Also in the Netherlands, $\sim 8000$ COPD-free subjects were followed with spirometry every 2-3 years, with additional follow-up in hospital and primary care and through pharmacy records. The study reported a $16 \%$ female and $24 \%$ male risk of developing COPD.

Although the above studies differ from ours, both the assumption of constant future age-specific rates and the basic principles of calculating lifetime risk are similar. However, due to the high frequency of fatal comorbidities and the slow progression of the disease, many patients with early stages of COPD will die from other causes before hospitalisation with COPD is required. Also, some patients develop fatal COPD, although they are never hospitalised with the disease. Hence, compared with the risks found in this present study, the aforementioned risks are higher, simply because the patients were diagnosed at earlier milder stages of the disease. The latter is illustrated by the lower mortality of, respectively, $5 \%$ and $6 \%$ in the studies by GERSHON et al. [6] and AFONSO et al. [8], respectively. Other major differences from our study include the fact that the studies by GERSHON et al. [6] and AFONSO et al. [8] excluded debuts of COPD after the age of 80 years, an approach that would have reduced our estimate by $\sim 25 \%$ (fig. 2 ). Also, the two latter studies used variable retrospective review periods of at least 1 year and at least 5 years, respectively. Reducing the retrospective review period would increase our lifetime risk estimate as demonstrated. Making it variable, e.g. from 8 years in 2002 to 14 years in 2008 , would decrease the risk estimate slightly, but would introduce a decreasing bias in the time trend. Finally, the GERSHON et al. [6] and VAN DuRME et al. [7] estimates are dated nearly one decade prior to ours and both the COPD incidence rates and mean life expectancies on which they are based probably have changed since then.

\section{Strengths and limitations}

The major strengths of this study include the size and duration of the study, the complete coverage of an entire nation, and the exact dates of hospitalisations, births and deaths. Also the homogenous equal access

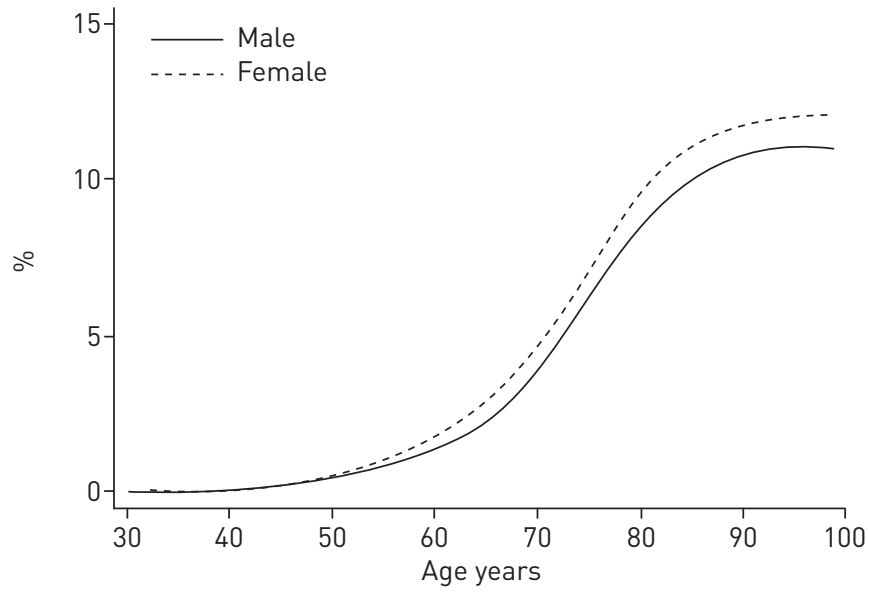

FIGURE 2 Accumulated incidence of first-time chronic obstructive pulmonary disease hospitalisation for 30-year-olds, adjusted for the competing risk of death. 
FIGURE 3 Residual lifetime risk of chronic obstructive pulmonary disease hospitalisation.

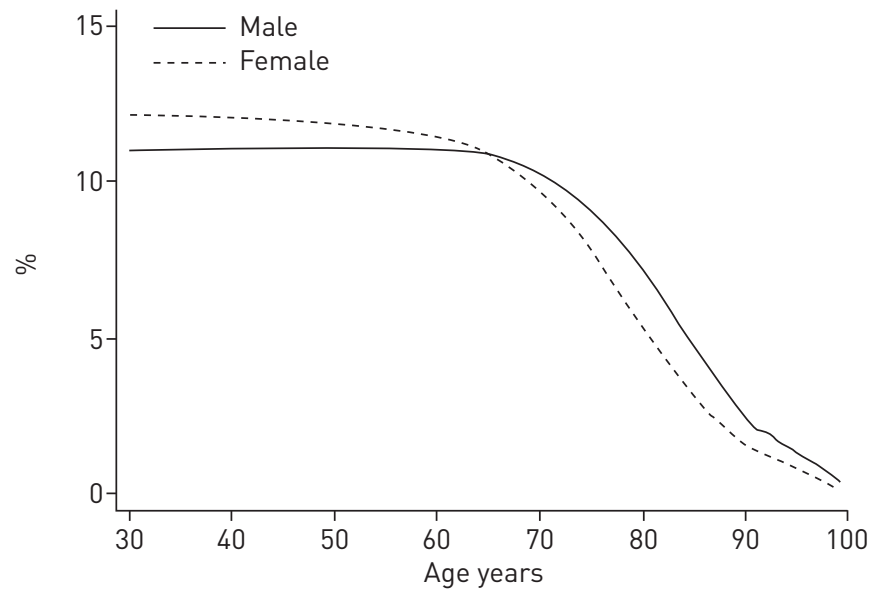

free-of-charge Danish Healthcare system supports equality in the decision criteria to hospitalise across the population.

The COPD diagnoses obtained from the registers were tested highly valid [14]. Nevertheless, misclassification remains a potential limitation of our study, and no spirometry data were available to further validate the diagnosis. Hence, our study includes some hospitalisations where the patients were wrongly diagnosed with COPD. However, it probably also failed to include even more hospitalisations that were caused by COPD, but miscoded with other diagnoses [14].

In large registers covering the general population, data on explaining factors, such as smoking status and spirometry results, do not exist. Furthermore, any conclusion as to the lifetime risk of, e.g. smoking, would require all the studied subjects to have maintained the same smoking status throughout the study period, and could be reasonably assumed to maintain that exact status until death or the occurrence of the event studied. Hence, regardless of study methods, for any risk factor besides sex, these requirements are nearly always impossible to meet.

The lifetime risk of COPD hospitalisation varies among populations because of different incidences of severe COPD exacerbations, different thresholds for admission to hospital, and different life expectancies. For decades the proportion of daily smokers has been higher in Denmark relative to other developed countries, especially among females [19]. Furthermore, the rate of COPD hospitalisations in Denmark [17] is somewhat higher than in contemporary studies from the USA [20-22], Norway, Sweden [23] and France [24]. Hence, compared with other developed countries, the lifetime risk of COPD hospitalisation is probably higher in Denmark. However, the mean life expectancy in Denmark is the same as in the USA, but shorter than in most European countries [19]. Shorter average lifespan means lower lifetime risk of all diseases mainly occurring in old age. Therefore, generally healthier populations in other countries may have even higher lifetime risks of COPD hospitalisation than in Denmark. The hospitalisation severity threshold probably differs between countries. However, characteristics of Danish COPD hospitalisations are in line

\section{TABLE 3 Age-specific residual lifetime risk of chronic obstructive pulmonary disease hospitalisation in Denmark}

\begin{tabular}{|c|c|c|c|c|c|c|}
\hline Age years & \multicolumn{3}{|c|}{ Females } & \multicolumn{3}{|c|}{ Males } \\
\hline 30 & $12.0(11.9-12.2)$ & $10.7(10.6-10.9)$ & 9.6 (9.5-9.7) & $10.9(10.8-11.1)$ & $9.4(9.3-9.6)$ & 8.4 (8.2-8.5) \\
\hline 40 & $12.0(11.9-12.1)$ & $10.7(10.6-10.8)$ & $9.6(9.5-9.7)$ & $11.0(10.9-11.1)$ & $9.4(9.3-9.6)$ & $8.4(8.3-8.6)$ \\
\hline 50 & $11.8(11.7-12.0)$ & $10.5(10.4-10.7)$ & $9.5(9.4-9.6)$ & $11.0(10.9-11.1)$ & $9.5(9.4-9.6)$ & $8.5(8.4-8.6)$ \\
\hline 80 & $5.3(5.1-5.4)$ & $4.5(4.4-4.6)$ & $4.0(3.9-4.1)$ & $7.0(6.9-7.2)$ & $5.8(5.6-5.9)$ & $5.1(5.0-5.3)$ \\
\hline 90 & $1.5(1.4-1.6)$ & $1.2(1.1-1.3)$ & $1.0(0.9-1.1)$ & $2.3(2.1-2.5)$ & $1.7(1.0-2.5)$ & $1.5(1.3-1.7)$ \\
\hline
\end{tabular}

Data are presented as $\%$ risk $(95 \% \mathrm{Cl})$. 
with other developed countries. For instance, the inpatient mortality, age, sex and length of stay regarding COPD hospitalisations are similar in Denmark, the USA and the UK $[9,17,25]$.

The main limitation of any lifetime risk study lies in the necessary assumption of time homogeneity of incidence and mortality rates [2,3]. Continuation of the increase in mean life expectancy, which has been steady for more than two centuries [19, 26], will increase the lifetime risk of COPD hospitalisation. Meanwhile, improved treatment possibilities and reductions in harmful airway exposure will decrease it.

In Denmark, during our observation period, the total number of COPD hospitalisations as well as the ageand sex-adjusted incidence rate of first-time cases decreased $\sim 8 \%$ [17]. In the same period the incidence and prevalence of hospitalisation-required COPD increased $\sim 20 \%$ in the age group $>80$ years while decreasing by $\sim 20 \%$ in the age group below. These large bidirectional age-specific trends have been explained as being due to the ageing of birth cohorts born around 1920, which were more exposed to both cigarette smoke [27] and occupational dust than the birth cohorts born before and after [28]. Also, the low exposure of the birth cohorts born before 1920 explains the observation from this study and others [8] that, in the oldest age group, COPD incidence actually decreases with increasing age. Furthermore, during our study period the ratio of patients treated with inhaled corticosteroids and tiotropium prior to their first COPD hospitalisation improved substantially [17]. It is likely that this had a reductive effect on COPD hospitalisation rates.

During our study period, there were some considerable changes in the Danish healthcare system. A $12 \%$ reduction in the number of hospital beds for COPD patients and at the same time fewer hospitalisations and increased inpatient mortality lead to the conclusion that the severity threshold for hospitalisation with COPD increased [17].

During the period from 2002 to 2008, in both sexes, the concurrent underlying mixture of increasing Danish overall life expectancy [19] and decreasing incidence rates of first-time COPD hospitalisations kept the lifetime risk of COPD hospitalisation constant. This balance is unlikely to remain stable. It is, however, unknown, whether increasing life expectancy or decreasing COPD incidence will be the stronger.

Estimates of mean life expectancy and lifetime risk are based on similar assumptions and thus have similar features. In most populations, known birth cohort effects make the mean life expectancy a poor estimate of the lifespan of a newborn. Likewise, the underlying changes in our study make the estimate for a 30-year-old a less valid predictor than that for an 80 -year-old. However, in a population, the mean life expectancy is considered a valid indicator of general health and similarly, the lifetime risk of a disease is perhaps the strongest and most robust indicator of its burden.

\section{Conclusion}

Based on present hospitalisation and survival rates, later in life $>10 \%$ of all 30 -year-old Danes will be hospitalised with COPD. Trends were generally equal in both sexes. During the period from 2002 to 2008, the rate of first-time COPD hospitalisations decreased, while the survival of never COPD hospitalised subjects increased. As a consequence, the lifetime risk of COPD hospitalisation remained constant.

\section{Acknowledgements}

We wish to thank secretary Lise Keller Stark (Research Unit of General Practice, Institute of Public Health, University of Southern Denmark, Odense, Denmark) for proofreading the manuscript.

\section{References}

Kohansal R, Martinez-Camblor P, Agusti A, et al. The natural history of chronic airflow obstruction revisited: an analysis of the Framingham offspring cohort. Am J Respir Crit Care Med 2009; 180: 3-10.

2 Keiding N. Age-specific incidence and prevalence - a statistical perspective. J Royal Statist Soc Series A 1991; 154: 371-412.

3 Sasieni PD, Shelton J, Ormiston-Smith N, et al. What is the lifetime risk of developing cancer? The effect of adjusting for multiple primaries. Br J Cancer 2011; 105: 460-465.

Berry JD, Dyer A, Cai X, et al. Lifetime risks of cardiovascular disease. N Engl J Med 2012; 366: 321-329.

Paap E, Broeders MJ, van Schoor G, et al. Large increase in a Dutch woman's lifetime risk of developing breast cancer. Eur J Cancer 2008; 44: 1485-1487.

6 Gershon AS, Warner L, Cascagnette P, et al. Lifetime risk of developing chronic obstructive pulmonary disease: a longitudinal population study. Lancet 2010; 378: 991-996.

7 van Durme YMTA, Verhamme KMC, Stijnen T, et al. Prevalence, incidence, and lifetime risk for the development of COPD in the elderly. Chest 2009; 135: 368-377.

8 Afonso AS, Verhamme KM, Sturkenboom MC, et al. COPD in the general population: prevalence, incidence and survival. Respir Med 2011; 105: 1872-1884. 
Price LC, Lowe D, Hosker HSR, et al. UK National COPD Audit 2003: impact of hospital resources and organisation of care on patient outcome following admission for acute COPD exacerbation. Thorax 2006; 61: 837-842.

10 Jansson SA, Andersson F, Borg S, et al. Costs of COPD in Sweden according to disease severity. Chest 2002; 122: 1994-2002.

11 Bastin AJ, Starling L, Ahmed R, et al. High prevalence of undiagnosed and severe chronic obstructive pulmonary disease at first hospital admission with acute exacerbation. Chron Respir Dis 2010; 7: 91-97.

12 Garcia-Aymerich J, Serra Pons I, Mannino DM, et al. Lung function impairment, COPD hospitalisations and subsequent mortality. Thorax 2011; 66: 585-590.

13 Statistics Denmark. Population in Denmark. www.dst.dk/en/Statistik/emner/befolkning-og-befolkningsfremskrivning/ folketal.aspx Date last accessed: April 25, 2013.

14 Thomsen RW, Lange P, Hellquist B, et al. Validity and underrecording of diagnosis of COPD in the Danish National Patient Registry. Respir Med 2011; 105: 1063-1068.

15 World Health Organization. International Classification of Diseases, 10th Revision. www.who.int/classifications/ icd/en/ Date last accessed: April 25, 2013.

16 Pedersen CB. The Danish Civil Registration System. Scand J Public Health 2011; 39: 22-25.

17 Lykkegaard J, Sondergaard J, Kragstrup J, et al. All Danish first-time COPD hospitalisations 2002-2008: incidence, outcome, patients, and care. Respir Med 2012; 106: 549-556.

18 Miravitlles M, Ferrer M, Pont A, et al. Effect of exacerbations on quality of life in patients with chronic obstructive pulmonary disease: a 2 year follow up study. Thorax 2004; 59: 387-395.

19 Organization for Economic Cooperation and Development Health Division. OECD Health Data 2006. www.oecd. org/dataoecd/20/51/37622205.xls Date last accessed: April 25, 2013.

20 Brown DW, Croft JB, Greenlund KJ, et al. Trends in hospitalization with chronic obstructive pulmonary diseaseUnited States, 1990-2005. COPD 2010; 7: 59-62.

21 Kabir Z, Connolly GN, Koh HK, et al. Chronic obstructive pulmonary disease hospitalization rates in Massachusetts: a trend analysis. QJM 2010; 103: 163-168.

22 Mannino DM, Homa DM, Akinbami LJ, et al. Chronic obstructive pulmonary disease surveillance - United States, 1971-2000. Respir Care 2002; 47: 1184-1199.

23 Liaaen ED, Henriksen AH, Stenfors N. A Scandinavian audit of hospitalizations for chronic obstructive pulmonary disease. Respir Med 2010; 104: 1304-1309.

24 Fuhrman C, Roche N, Vergnenegre A, et al. Hospital admissions related to acute exacerbations of chronic obstructive pulmonary disease in France, 1998-2007. Respir Med 2011; 105: 595-601.

25 American Lung Association. The Scope of COPD in Illinois - Executive Summary. www.lung.org/associations/ states/illinois/assets/pdfs/scope-of-copd-summary-2011.pdf Date last accessed: April 25, 2013. Date last updated: March 2011.

26 Christensen K, Doblhammer G, Rau R, et al. Ageing populations: the challenges ahead. Lancet 2009; 374: 1196-1208. Madsen M, Videbæk J. Heart Statistics 2004. Copenhagen, Danish National Institute of Public Health, 2004.

Lykkegaard J, Davidsen JR, Paulsen MS, et al. On the crest of a wave: Danish prevalence of hospitalisation-required COPD 2002-2009. Respir Med 2012; 106: 1396-1403. 